\title{
Absence of Chaos in Bohmian Dynamics
}

\author{
Sheldon Goldstein* \\ Department of Mathematics, Rutgers University, Piscataway, NJ 08854-8019
}

(January 5, 1999)

\begin{abstract}
The Bohm motion for a particle moving on the line in a quantum state that is a superposition of $n+1$ energy eigenstates is quasiperiodic with $n$ frequencies.

PACS number(s): 05.45.+b, 03.65.Bz, 03.65.Ge
\end{abstract}

In a recent paper [1], O. F. de Alcantara Bonfim, J. Florencio, and F. C. Sa Barreto claim to have found numerical evidence of chaos in the motion of a Bohmian quantum particle in a double square-well potential, for a wave function that is a superposition of five energy eigenstates. But according to the result proven here, chaos for this motion is impossible. We prove in fact that for a particle on the line in a superposition of $n+1$ energy eigenstates, the Bohm motion $x(t)$ is always quasiperiodic, with (at most) $n$ frequencies. This means that there is a function $F\left(y_{1}, \ldots, y_{n}\right)$ of period $2 \pi$ in each of its variables and $n$ frequencies $\omega_{1}, \ldots, \omega_{n}$ such that $x(t)=F\left(\omega_{1} t, \ldots, \omega_{n} t\right)$.

The Bohm motion for a quantum particle of mass $m$ with wave function $\psi=\psi(x, t)$, a solution to Schrödinger's equation, is defined by

$$
d x / d t=(\hbar / m) \operatorname{Im} \nabla \psi / \psi
$$

The right hand side of (11) depends upon $\psi$ only through its associated ray. In particular, if the wave function

$$
\psi(x, t)=\Sigma_{i=0}^{n} a_{i} e^{-i E_{i} t / \hbar} \phi_{i}(x)
$$

is a superposition of $n+1$ energy eigenstates $\phi_{i}$, then the right hand side of (11) is, in its dependence upon $t$, quasiperiodic with $n$ frequencies, as is $|\psi|$.

The quasiperiodicity in time of the vector field defining a dynamical system in general does not imply any corresponding property of the motion, since an autonomous system (one defined by a time independent vector field) can be chaotic. (In fact, it is autonomous systems that are normally studied in chaos theory.) However, for the Bohm motion on the line, the position of the particle is anchored in the (normalized) wave function, in such a way that its motion $x(t)$ inherits the quasiperiodicity of $|\psi|:$

A crucial feature of the motion (11) is the equivariance of $|\psi|^{2}$, i.e., the fact that probabilities for configurations given by $|\psi(x, t)|^{2}$ are consistent with the dynamics (1). This is a completely general feature of the Bohmian dynamics, valid in any dimension for any wave function satisfying Schrödinger's equation. For a single particle moving on the line, it has the following important consequence:

\footnotetext{
*Electronic address: oldstein@math.rutgers.edu
}

$$
\int_{-\infty}^{x(t)}\left|\psi\left(x^{\prime}, t\right)\right|^{2} d x^{\prime}=\int_{-\infty}^{x(0)}\left|\psi\left(x^{\prime}, 0\right)\right|^{2} d x^{\prime}
$$

which follows from equivariance since in one-dimension the dynamics is order-preserving, and in particular the evolution from time 0 to time $t$ carries the interval $(-\infty, x(0))$ to $(-\infty, x(t))$.

Given $\psi(x, 0)$ and $x(0)$, equation (3) determines $x(t)$ as a functional of $|\psi(x, t)|^{2}$, and thus $x(t)$, like $|\psi(x, t)|^{2}$, is quasiperiodic with $n$ frequencies. In fact $x(t)=$ $F\left(\omega_{1} t, \ldots, \omega_{n} t\right)$ with $\omega_{i}=\left(E_{i}-E_{0}\right) / \hbar$ for $i=1, \ldots, n$ and $F\left(y_{1}, \ldots, y_{n}\right)=G\left(\int_{-\infty}^{x(0)}\left|\psi\left(x^{\prime}, 0\right)\right|^{2} d x^{\prime}\right)$ where $G$ is the inverse of the function $H(x)=\int_{-\infty}^{x}\left|\psi\left(x^{\prime}\right)\right|^{2} d x^{\prime}$ with

$$
\psi(x) \equiv \psi_{y_{1}, \ldots, y_{n}}(x)=a_{0} \phi_{0}(x)+\sum_{i=1}^{n} a_{i} e^{-i y_{i}} \phi_{i}(x) .
$$

For the one-dimensional motion $x(t)$ the Lyapunov exponent $\lambda$ is given by

$$
\lambda=\lim _{t \rightarrow \infty} t^{-1} \ln \frac{d x(t)}{d x(0)} .
$$

It presumably follows from the quasiperiodicity of $x(t)$ alone that $d x(t) / d x(0)$ is similarly quasiperiodic. In any case, we have by equivariance that $|\psi(x(t), t)|^{2} d x(t)=$ $|\psi(x(0), 0)|^{2} d x(0)$, so that

$$
d x(t) / d x(0)=\frac{|\psi(x(0), 0)|^{2}}{\left|\psi_{y_{1}, \ldots, y_{n}}\left(F\left(y_{1}, \ldots, y_{n}\right)\right)\right|^{2}}
$$

with $y_{i}=\omega_{i} t$. Hence $d x(t) / d x(0)$ is quasiperiodic with $n$ frequencies and thus $\lambda=0$.

Remarks: (i) In one-dimension we always have that $d x(t) / d x(0)=|\psi(x(0), 0)|^{2} /|\psi(x(t), t)|^{2}$. Thus the vanishing of the Lyapunov exponent $\lambda$ is more general than described here, and should be valid for any wave function, on the circle as well as the line. After all, for bound states the ratio on the right is not likely to grow or decrease in any systematic way at all, while for states with continuous spectrum the behavior will be at most power law; in no case will there be exponential growth or decay. (ii) Another aspect of chaos, the weak convergence of densities to the "equilibrium" distribution (for Bohmian mechanics given by $|\psi(x(t), t)|^{2}$ ) will, as a simple consequence of the order preserving character of such motions, almost always fail for any one-dimensional flow, Bohmian or otherwise. The sole exception can occur only when the asymptotic "equilibrium" distribution is concentrated on a single (perhaps moving) point, something that is impossible for Bohmian mechanics.

I am grateful to Michael Kiessling for helpful suggestions. This work was supported in part by NSF Grant No. DMS-9504556. 
[1] O. F. de Alcantara Bonfim, J. Florencio, and F. C. Sá Barreto, Phys. Rev. E 58, 6851 (1998). 